\title{
Video Article \\ Determining heat and mechanical pain threshold in inflamed skin of human subjects
}

\author{
Martin S Angst ${ }^{1}$, Martha Tingle ${ }^{1}$, Nicholas G Phillips ${ }^{1}$, Brendan Carvalho ${ }^{1}$ \\ ${ }^{1}$ Department of Anesthesia, Stanford University School of Medicine
}

URL: https://www.jove.com/video/1092

DOI: doi:10.3791/1092

Keywords: Medicine, Issue 23, Experimental pain, experimental inflammation, human, skin, heat stimuli, mechanical stimuli, pain threshold, psychophysics, non-myelinated nociceptive nerve fiber, small myelinated nociceptive nerve fiber

Date Published: 1/14/2009

Citation: Angst, M.S., Tingle, M., Phillips, N.G., Carvalho, B. Determining heat and mechanical pain threshold in inflamed skin of human subjects. J. Vis. Exp. (23), e1092, doi:10.3791/1092 (2009).

\section{Abstract}

In a previous article in the Journal of Visualized Experiments we have demonstrated skin microdialysis techniques for the collection of tissuespecific nociceptive and inflammatory biochemicals in humans. In this article we will show pain-testing paradigms that are often used in tandem with microdialysis procedures. Combining pain tests with microdialysis provides the critical link between behavioral and biochemical data that allows identifying key biochemicals responsible for generating and propagating pain.

Two models of evoking pain in inflamed skin of human study participants are shown. The first model evokes pain with aid of heat stimuli. Heat evoked pain as described here is predominantly mediated by small, non-myelinated peripheral nociceptive nerve fibers ( $\mathrm{C}$-fibers). The second model evokes pain via punctuated pressure stimuli. Punctuated pressure evoked pain is predominantly mediated by small, myelinated peripheral nociceptive nerve fibers (A-delta fibers). The two models are mechanistically distinct and independently examine nociceptive processing by the two major peripheral nerve fiber populations involved in pain signaling.

Heat pain is evoked with aid of the TSA II, a commercially available thermo-sensory analyzer (Medoc Advanced Medical Systems, Durham, NC). Stimulus configuration and delivery is handled with aid of specific software. Thermodes vary in size and shape but in principle consist of a metal plate that can be heated or cooled at various rates and for different periods of time. Algorithms assessing heat-evoked pain are manifold. In the experiments shown here, study participants are asked to indicate at what point they start experiencing pain while the thermode in contact with skin is heated at a predetermined rate starting at a temperature that does not evoke pain. The thermode temperature at which a subject starts experiencing pain constitutes the heat pain threshold.

Mechanical pain is evoked with punctuated probes. Such probes are commercially available from several manufacturers (von Frey hairs). However, the accuracy of von Frey hairs has been criticized and many investigators use custom made punctuated pressure probes. In the experiments shown here eight custom-made punctuated probes of different weights are applied in consecutive order, a procedure called updown algorithm, to identify perceptional deflection points, i.e., a change from feeling no pain to feeling pain or vice versa. The average weight causing a perceptional deflection constitutes the mechanical pain threshold.

\section{Video Link}

The video component of this article can be found at https://www.jove.com/video/1092/

\section{Heat Pain Testing using the Thermostimulator TSA II}

\section{Introduction}

1. This procedure uses the TSA II, a commercially available thermo-sensory analyzer that delivers heat stimuli to the skin with a thermode (Medoc Advanced Medical Systems, Durham, NC). A central unit generates and transmits the signal to the thermode. This signal is controlled by proprietary software typically stored on a laptop connected to the central unit. Also attached to the central unit is a hand-held control that allows a study subject to stop the heating of the thermode.

2. Seven sequential and identical heat stimuli are delivered, with inter-stimulus intervals of $30 \mathrm{~s}$. The basal thermode temperature is set at a comfortable $35^{\circ} \mathrm{C}$. The rate at which the thermode heats up is set at $1{ }^{\circ} \mathrm{C} / \mathrm{s}$, while the rate at which it cools down is set at $10^{\circ} \mathrm{C} / \mathrm{s}$. The maximum thermode temperature is set at $52^{\circ} \mathrm{C}$. 


\section{Preparation and Training the Subjects}

1. To prepare subjects for the heat pain test seat them comfortably in a reclining chair that provides adequate support for the head, arms and legs. The chair should also allow easy access to the examined body sites.

2. The testing facility should be at a comfortable room temperature, and provide a quite and neutral environment with no distraction.

3. Before starting the experiment, all participants should be well trained in all aspects of the heat pain testing. Training can take place any time before the experiment.

4. Interactions between study subjects and investigators should be limited to those necessary for collecting data. Joking and small talk are discouraged because they may compromise data consistency. Study subjects are informed that interactions will intentionally be somewhat monotonic and neutral during the experiments.

5. Keep the instructions clear, simple, and consistent. Emphasize that pain is subjective, and there are no right or wrong answers. It is most important to be consistent. Define the heat pain threshold as the point between being "about to be painful" and "just became painful."

6. It is critical that a subject knows precisely what to expect during a test session. Any "surprises" may result in less consistent data, because confounders such as anxiety may be generated. For example, if seven heat pain stimuli are used to determine the heat pain threshold, the subject should know this.

7. At any time, the subject can stop the thermode from getting any hotter by pushing the button of a hand-held control. Being in control will reduce a subject's anxiety and will make the data more reliable.

8. The training session offers an opportunity to judge a study subject's performance. It may be best to train subjects during two to four test cycles so their individual pain thresholds vary by no more than $0.8^{\circ} \mathrm{C}$, and ideally by no more than $0.6^{\circ} \mathrm{C}$ between the last two test cycles. Subjects who fail to perform consistently are typically excluded from the study.

\section{Determining the Pain Threshold}

1. To begin the pain threshold testing cycles mark the placement of the thermode on the subject's skin. In this experiment the same inflamed skin lesion used for microdialysis will be tested for pain to learn about biochemicals released in response to the pain testing.

2. Then, initiate each cycle by placing the thermode onto the marked skin area. Match the position of the thermode with the markings as closely as possible. Allow the temperature between the skin and the thermode to equilibrate to $35^{\circ} \mathrm{C}-\mathrm{a}$ temperature that safely will cause no pain. This will take about 5 seconds and will be indicated by a reading of $35^{\circ} \mathrm{C}$ on the computer screen.

3. Before beginning, give the hand-held control to the subject.

4. Use constant and gentle pressure to keep the entire surface of the thermode in full contact with the subject's skin during testing.

5. Now start the pre-programmed test algorithm. The thermode will start heating up at $1^{\circ} \mathrm{C} / \mathrm{s}$. As soon as the subject experiences pain, they'll press the hand held device.

6. After the button press on the hand-held device, the thermode temperature causing pain will automatically be registered and will start decreasing at a rate of $10^{\circ} \mathrm{C} / \mathrm{s}$ down to its baseline temperature of $35^{\circ} \mathrm{C}$.

7. The temperature probe will automatically be kept at $35^{\circ} \mathrm{C}$ for $30 \mathrm{~s}$ before it starts rising again at $1^{\circ} \mathrm{C} / \mathrm{s}$, until a subject again pushes the button of the hand-held device. Just before the thermode temperature is about to increase again, remind the subject that this is about to happen. This will keep the subject focused.

8. This sequence will be repeated until seven temperature recordings have been registered. Calculate the average of these temperature readings; this is the heat pain threshold.

\section{Mechanical Pain Testing with Punctuated Pressure Probes}

\section{Introduction}

1. We use custom made calibrated punctuated pressure probes, because they provide more consistent results than the traditionally used and commercially available von Frey hairs. Probes are made of identical cylindrical stainless steel wires ( $240 \mu \mathrm{m}$ diameter $)$ mounted on copper rods of various weights $(1,2,4,8,16,32,49,64$, and 81 grams) that move freely within a wider hand-held aluminum tube.

2. The same general experimental conditions outlined for heat pain testing apply to mechanical pain testing. The subjects should be well prepared and trained. It is especially important to reassure subjects that the probes are blunt and will not puncture the skin. This will help the subjects to relax and provide consistent data.

\section{Determining the Mechanical Pain Threshold}

1. To determine the mechanical pain threshold, first apply the steel wire tip of the lightest probe perpendicularly to the skin at the center of the experimentally inflamed lesion.

2. The hand-held aluminum tube is then lowered towards skin without touching it. This way, the entire weight of the copper rod inside the aluminum tube rests on the steel wire tip.

3. The study subject then indicates whether the probe causes pain by saying "yes" or "no."

4. If the subject answers "no," then either the same probe or next heavier probe will be used next. This algorithm is called an ascending stimulation sequence. Randomly determine whether the next probe is heavier or of the same weight. This will keep a subject blinded to the algorithm.

5. Apply the probes in ascending sequence until a subject answers "yes." The average weight of the last two probes in this series is recorded as the first deflection point.

6. After the subject answers "yes," start using a descending stimulation sequence, and use either a lighter probe or the same probe as before. Randomly determine whether the next probe is lighter or of the same weight. This will keep a subject blinded to the algorithm. 
7. Apply probes in a descending sequence until the subject answers "no." The average weight of the last two probes in this sequence constitute the second deflection point.

8. When the subject answers "no," switch back to an ascending stimulation sequence. Alternate between ascending and descending sequences until seven deflection points are recorded. The average of these seven points is the mechanical pain threshold.

\section{Discussion}

This video demonstrates algorithms for assessing the heat and mechanical pain threshold in inflamed skin of human volunteers. The perception of pain is subjective. Collection of adequate pain data requires an appropriate testing facility (e.g. quiet and comforting), thorough training of study participants with in-built data quality control measures (test-retest reliability), strict adherence to a standardized study protocol, and avoidance of confounding factors (e.g. stress, anxiety). Reliable subjective data on pain sensitivity, paired with interventions such as the administration of an analgesic drug and the collection of mechanistic data such as the local release of nociceptive mediators provide valuable insight into mechanisms of pain, and means to prevent or attenuate it.

\section{References}

1. Angst, M.S., Clark, J.D. Comment on Koltzenburg et al.: Differential sensitivity of three experimental pain models in detecting the analgesic effects of transdermal fentanyl and buprenorphine. Pain. 126, 165-74, (2006). Pain. 128, 292-4, (2007).

2. Angst, M.S., Clark, J.D., Carvalho, B., Tingle, M., Schmelz, M., Yeomans, D.C. Cytokine profile in human skin in response to experimental inflammation, noxious stimulation, and administration of a COX-inhibitor: A microdialysis study. Pain. (2008).

3. Angst, M.S., Koppert, W., Pahl, I., Clark, J.D., Schmelz, M. Short-term infusion of the mu-opioid agonist remifentanil in humans causes hyperalgesia during withdrawal. Pain. 106, 49-57 (2003).

4. Lotsch, J., Angst, M.S. The mu-opioid agonist remifentanil attenuates hyperalgesia evoked by blunt and punctuated stimuli with different potency: a pharmacological evaluation of the freeze lesion in humans. Pain. 102, 151-61 (2003). 